\title{
Impact of Multiscale Mineralogical and Sedimentary Heterogeneity on Mechanical Behavior of Mancos Shale
}

\author{
HONGKYU YOON ${ }^{1}$
}

Geomechanics Department, Sandia National Laboratories, NM, USA

1hyoon@sandia.gov

The performance of unconventional resources reservoir is a function of the hydro, mechanical, and chemical properties of shale formations with compositional and textural heterogeneity across a range of scales. In particular, mechanical properties (elastic properties, fracture toughness, anisotropy, etc.) are controlled by a variety of geologic variables, including mineralogy, cements, and organic content, and the spatial distribution of these characteristics. In this work an integrated approach of multiscale imaging, mineralogy distribution, nano-indentation, machine learning, sedimentation features, and numerical simulations is employed to investigate the impact of the micro-lithofacial heterogeneities on mechanical properties for Cretaceous Mancos Shale.

Nano-indentation results are mapped into detailed mineralogical distribution based on MAPS (Modular Automated Processing System) technique and high resolution backscattered electron images to relate measured mechanical properties to depositional characteristics of micro-lithofacies. Numerical simulation result of nano-indentation is used to interpret the impact of spatial heterogeneity of composition, texture phases, and interfaces of phases on mechanical responses. A range of Young's moduli from nano-indentation is generally larger by a factor of 1 to 4 compared to axisymmetric compression results, showing the important effect of pores, microcracks, and bedding boundaries on bulk elastic response. Together these data sets show the influence of cement distribution on mechanical response. Variations in micro-lithofacies are first-order factors in determining the mechanical response of this important Mancos constituent, and are likely responsible for its success in hydrofracturebased recovery operations as compared to other Mancos lithofacies types.

Sandia National Laboratories is a multimission laboratory managed and operated by National Technology and Engineering Solutions of Sandia, LLC., a wholly owned subsidiary of Honeywell International, Inc., for the U.S. Department of Energy's National Nuclear Security Administration under contract DE-NA-0003525. 